\title{
Variations
}

Variations

Revue internationale de théorie critique

$24 \mid 2021$

Echos

\section{Débat à propos du nouveau manifeste}

Traducteur : David Buxton

\section{(2) OpenEdition}

Journals

Édition électronique

URL : https://journals.openedition.org/variations/2018

DOI : 10.4000/variations.2018

ISSN : 1968-3960

Éditeur

Les amis de Variations

Référence électronique

"Débat à propos du nouveau manifeste », Variations [En ligne], 24 | 2021, mis en ligne le 03 juillet 2021 , consulté le 07 juillet 2021. URL : http://journals.openedition.org/variations/2018 ; DOI : https:// doi.org/10.4000/variations.2018

Ce document a été généré automatiquement le 7 juillet 2021.

Les ami•e•s de Variations 


\section{Débat à propos du nouveau manifeste}

Traduction : David Buxton

1 Nicholas Brown (modérateur): Il me semble que les trois interventions soulèvent essentiellement la même question, mais non explicitement. Alors, c'est cette question que je vais vous poser. J'avoue que je n'ai pas pu terminer le dialogue entre Adorno et Horkheimer, justement à cause de son atmosphère beckettienne. De toute évidence, ils se retrouvent face à une impossibilité, à savoir comment rester fidèle à Lénine sans parti, sans affiliation viable, sans concept de parti opératoire. Bien entendu, la question devient ainsi : que faire quand il n'y a rien à faire?

2 On trouve une version tragique de cela dans La Dialectique négative où Adorno, en connaissance de cause, rejoint les Stoïques, et présente sa propre position comme essentiellement stoïque, sachant mieux, ou du moins aussi bien que quiconque que l'entière force éthique de La Phénoménologie de l'esprit, qu'hérite Marx, est l'impossibilité de la position stoïque.

3 L'effacement délibéré du langage dans le dialogue entre Adorno et Horkheimer ressemble à la conscience malheureuse dans La Phénoménologie de l'esprit, qui oscille précisément pour la même raison que chez eux. Leur conscience malheureuse est incapable à saisir « l'anneau suivant » (Lukács), car celui-ci n'existe pas - ce qui pose encore le problème du parti.

4 Cela nous amène à la question du « parti » chez Lukács. Voici ma question pour Andrew [Feenberg] : que faisons-nous - que faire? - sans parti? Tu sembles suggérer que la réponse se retrouve chez Marcuse.

5 Richard [Westermann] montre que, pour Lukács, « le parti » n'est pas tant une chose, nécessairement, qu'un concept. Le parti est cette chose qui médie entre le sujet et l'Histoire. Le moment qu'on nie l'épistémologie, le moment qu'on nie l'ontologie, le moment qu'on nie Kant, le moment qu'on nie la représentation, alors, on se retrouve dans un univers hégélien où il y a l'obligation de trouver "le parti », "l'anneau suivant ", ou "la médiation ». Voilà l'obligation qu'Adorno est incapable à honorer; c'est à la fois sa tragédie et sa farce. Alors, ma question pour toi, Richard, est la même : à quoi ressemble le concept philosophique du parti aujourd'hui? Tu nous donnes une 
sorte de réponse autonomiste, negrienne, qui ne me semble pas satisfaisante, car c'est comme si Hegel attendait Hardt et Negri aussi. Comme si le sujet était une fiction, mais une fiction nécessaire - un peu comme un parti est nécessaire.

6 Pour Chris [Cutrone], il semble que chez Marx, chez Lukács, et certainement chez Adorno et Marcuse, il y ait une tension non résolue entre la notion de non-liberté universelle et la notion d'exploitation. Cette dernière, dans notre époque, renvoie à l'idée de fragilité ; qui est, et qui n'est pas protégé des vents de l'Histoire ? Ce n'est pas exactement la même question que celle de la non-liberté universelle, et de la désaliénation.

7 C'est la notion de désaliénation, le côté romantique des éclats chez Marx, Lukács, et l'École de Francfort, qu'il faut abandonner, à mon avis, en faveur d'un accent plus réaliste sur l'exploitation. Si, pour l'École de Francfort, l'idéal était le prochain pas ou l'anneau suivant, que veut dire l'idée hégélienne actuellement?

8 Andrew Feenberg : Ce que j'aime bien chez Marcuse, c'est qu'il a réussi à séparer deux choses qui chez Marx, Lukács et Lénine sont essentiellement connectées. L'une est le sujet de la révolution, et l'autre est la force capable de déréifier au moins une partie de la réalité sociale. Dans la conception marxiste classique, ce sont les ouvriers qui déréifient, par leur refus de se soumettre passivement aux formes dans lesquelles leurs vies sont fondues; ce sont aussi les ouvriers qui vont créer la société nouvelle. Marcuse s'est rendu compte qu'on peut avoir l'un sans l'autre. On peut exprimer sa solidarité avec des gestes déréifiants, les articuler théoriquement, sans être du tout certain que les auteurs de ces gestes soient capables de renverser la société et en créer une nouvelle. Après les évènements de mai '68 en France, il était clair qu'un type d'opposition radicalement nouveau avait surgi. Je pense que Marcuse avait raison d'essayer de relier la théorie de Marx à cette opposition. C'est une alternative significative au désespoir d'Adorno et de Horkheimer, et de l'autre côté, aux tentatives de relancer un parti marxiste traditionnel.

Richard Westermann : Ma réponse à "que faire?» est que ce n'est pas à nous de répondre. Je pense que cela eût été la réponse de Lukács. Le parti (ou toute forme d'organisation), plutôt que d'être un instrument, devrait être perçu comme la façon dont de multiples volontés apprennent à se penser comme une unité (et pas nécessairement comme une entité). Pas tant pour les décisions spécifiques par lesquelles on en vient à la pratique, mais plutôt pour l'auto-organisation, pour les formes institutionnelles qu'on se donne.

10 Je pense que la critique lukácsienne de Hegel (et en effet, de la philosophie bourgeoise en général) a son origine dans l'idée d'un sujet : l'idée qu'il faudrait concevoir l'action comme un sujet agissant sur le monde et reconnaissant lui-même. Ce que Lukács voit dans le parti, c'est une entité (si je peux utiliser un terme ontologiquement réifiant), qui est un sujet en ce qu'elle se manifeste objectivement dans ses formes d'organisation. Ce n'est pas tout à fait la même chose que de concevoir le parti comme un agent.

11 Chris Cutrone : Ce que nous discutons ici, c'est la forme politique. Autrement dit, le parti est une forme. Nous parlons du parti comme une forme de médiation : celle de la théorie et de la pratique, et celle des positions du sujet et de l'objet.

12 Quant à l'idéal hégélien comme le prochain pas pour Adorno et Horkheimer, j'en parlerai de façon spéculative, et non littérale. Andrew [Feenberg] a noté l'ambiguïté 
fondamentale du Marx tardif par rapport à la façon dont il conçoit la philosophie dans sa jeunesse. J'affirmerais que la question de médiation est récurrente. La critique de l'économie politique n'est pas seulement une analyse des formes «bourgeoises ", mais aussi une analyse critique de la conscience naissante du mouvement ouvrier. Celui-ci hérite l'économie politique, la conscience sociale bourgeoise, mais seulement quand la pensée de la bourgeoisie elle-même devient vulgaire. Marx loue Adam Smith pour sa volonté de présenter la société comme contradictoire en elle-même. Alors, je mettrais en relation la question du prochain pas avec celle de la critique du capital. Comment réarticuler la praxis politique propre de Marx avec sa critique théorique du capital ? Cette critique était une tentative hégélienne d'élever la forme sociale au niveau de la conscience de soi pour les militants de la classe ouvrière, qui se retrouvaient face à certains obstacles très déterminants pour leur pratique politique dans le sillage des révolutions de 1848. Il y eut une "rencontre», pour adopter les termes plus traditionnels d'Adorno, entre des intellectuels et des ouvriers autour de la question de ce qu'on pouvait faire avec la critique du capital.

13 Après les années 1960, il y a eu un retour à Marx, un retour au marxisme hégélien à l'égard de la critique du capital. Si nous nous décrivions comme intellectuels, alors la question à nous poser serait: "comment ces idées peuvent-elles avoir un effet»? Korsch dit que la crise du marxisme menace de rompre le cordon ombilical entre théorie et pratique ; cela implique qu'elles soient deux choses séparées. Pour ma part, je soulignerais la médiation dans le concept de forme par rapport à la liquidation de la théorie et de la pratique dans le concept de parti.

\section{Réponses aux questions du public}

QUESTION : Si nous autres, marxistes, communistes, ou révolutionnaires aspirant(e)s, ne sommes pas dans une position de parler, alors nous devrions nous demander: que faudrait-il pour nous transformer en ceux ou celles qui pourraient parler? Comment pouvons-nous écrire comme Lénine ou Mao? J'ai été frappé(e) par le dialogue Adorno/ Horkheimer; Horkheimer n'est pas seul en attribuant les morts provoquées par le Grand Bond en avant à Mao et à Staline. Que serait-il passé si, au lieu de lancer des bouteilles à la mer, Horkheimer et Adorno avaient envoyé leurs messages en Chine, et n'avaient pas tiré une croix prématurément à cette révolution réelle?

RW : Il n'y a pas d'interdiction à « parler » en tant que telle. Mais cela dépend de si on parle ex cathedra ou au sein d'une autre structure. Je suis d'accord avec Habermas quand il insiste que pour parler de telles choses, il faut se mettre à un pied d'égalité avec tout le monde. C'est un danger que Lénine lui-même reconnaît, dans ses lettres finales furieuses demandant que le parti s'éloigne autant que faire se peut des soviets, affirmant que les ouvriers et les paysans honnêtes seraient soit trop intimidés, soit trop respectueux devant les sages de Moscou. Ce qu'il faut faire pour pouvoir parler, alors, c'est nier ce que nous sommes ; s'il en existe un danger, c'est toujours celui-ci, à mon avis, pour quelqu'un qui parle avec le moindre badge d'autorité. Cela mène à ce genre de problème de direction intellectuelle, où justement la liberté envisagée par Marx et par d'autres est mise sur la touche.

AF : Je ne suis pas d'accord! Il n'existe plus de paysans ignorants. Ceux qui s'opposent à l'autorité intellectuelle le plus bruyamment sont eux-mêmes intellectuels. Alors, c'est juste une autre théorie ! Je ne savais pas qu'il y avait là un problème ; pour moi, c'est plutôt une question de savoir s'il y a encore quelqu'un qui 
veut nous écouter, et non si nous sommes oppressifs en voulant présenter nos points de vue. Voilà ma conclusion, après avoir participé dans les bons vieux temps à beaucoup de luttes sur la question de l'autorité.

CC : S'agissant de la transformation des intellectuels par eux-mêmes, le problème n'est pas de qui parle, mais de ce qui est dit. J'ai envie d'introduire ici une autre catégorie léniniste, à savoir le suivisme. Articuler la conscience historique et les réalités empiriques pose problème. J'aimerais retourner à une question utile soulevée par Andrew et par Richard à l'égard de la réification. Ce que Lukács voulait dire par réification, c'était la Deuxième Internationale, le mouvement ouvrier socialiste tel qu'il s'était constitué dans la conjoncture historique. S'il est favorable à Luxemberg, c'est parce qu'elle critique la forme-parti dans son pamphlet Grève de masse, où elle affirme que la social-démocratie est devenue un obstacle au mouvement ouvrier dans, je dirais, une dialectique sujet-objet: le mouvement ouvrier s'est généré historiquement en objet d'une autocritique.

Or, Horkheimer avait peur de la Chine en raison du succès "révolutionnaire » apparent de ce que lui et Adorno considéraient comme la contre-révolution, à savoir le stalinisme. Ayant vécu dans les années 1930 quand le marxisme se transformait en stalinisme, ils ne pouvaient qu'appréhender le fleurissement du stalinisme dans la période d'après-guerre comme le signe d'une régression du marxisme lui-même. Alors, pourquoi n'envoyaient-ils pas leurs messages à des intellectuels en Chine? Parce que ça aurait été la meilleure façon d'assurer l'exécution de ces intellectuels sur-le-champ. Il est possible de lire leurs affirmations comme la preuve d'un biais antichinois prime facie. Mais il y a une dialectique là. Comme le demande Horkheimer, que dire si 20 millions de Chinois vont mourir, mais qu'après il n'y avait plus d'affamés? Comment interpréter cela? Ce qu'avaient à l'esprit Horkheimer et Adorno, c'est que si le succès de la révolution russe en 1917 avait gagné l'Allemagne et au-delà, une révolution en Chine en 1949, avec tous les sacrifices et toutes les calamités qu'elle a entraînés, n'aurait pas été nécessaire. C'était cela, leur vision de l'émancipation ; leur souci, c'était que le barbarisme se confondait avec la lutte pour l'émancipation.

NB : Quand il existe un mouvement de masse, la situation de l'intellectuel est à la fois beaucoup plus facile et beaucoup plus difficile. Il est facile parce que vous savez que faire, mais le projet de transformation que vous défendez est difficile. Le problème que nous affrontons aujourd'hui est différent : il n'y a pas de mouvement de masse. Et dans la mesure où il y en a un, il est totalement corrompu et droitier.

Très clairement, Adorno s'aligne sur l'Occident, donc la question de sa lecture par des dissidents chinois est moins pertinente que celle-ci : est-ce qu'Adorno aurait dû s'aligner à ce point-là sur l'Occident, et donc couper si nettement les liens avec le socialisme réellement existant? Cette question est un peu moins évidente que de demander s'il eût été salutaire d'avoir des dissidents chinois reprenant les arguments adorniens.

QUESTION : Kant nous demande de penser politiquement, en ce que nous sommes obligés de commenter sur la société en tant que membres de cette même société; nous sommes obligés de contribuer au développement de la société. Lukács a perçu que c'est seulement au travers d'un parti que la société peut continuer à développer, alors la question de la responsabilité individuelle dans l'Histoire me semble quelque peu déplacée. C'est seulement le parti qui, ayant la capacité à façonner l'Histoire, est obligé de réfléchir sur 
I'histoire. Se peut-il que ce soit cela qui motive Lénine et Luxemberg quand ils parlent du parti? C'est-à-dire, quand Luxemberg s'inquiète du vote au Reichstag sur les crédits de guerre, son vrai souci est le déclin du parti, et le besoin de le reconfigurer afin d'influer sur le cours de l'histoire.

RW : Je ne suis pas d'accord. Lukács ne pense pas que le parti puisse changer l'Histoire, c'est la classe qui peut le faire. Le parti réalise la classe. Le parti est peutêtre le point de départ, mais catégoriquement n'est pas le point d'aboutissement. Dire que le parti change l'Histoire directement lui donnerait une sorte de rôle héroïque que Lukacs essaie d'éviter à mon avis.

CC : Je dirais que le parti politique, ou l'agent de médiation politique ne peut, en luimême, émanciper la société. Mais il peut certainement bloquer cette émancipation, et être perçu de façon négative. L'importance du parti dépend de la question de conscience historique. Alors, là où j'ai plus de sympathie pour la critique que Luxemberg fait du SPD lors de son effondrement politique, c'est son affirmation que le parti est responsable devant l'Histoire, de façon négative. Elle dit que le parti a joué un rôle en poussant l'Histoire à un point critique, et que désormais c'est la tâche du parti de se surmonter sous sa forme d'agence politique médiatrice.

QUESTION : D'abord, je trouve que le Lénine que vous décrivez - médié par Adorno et par Lukács - est totalement méconnaissable du Lénine des œuvres complètes. Le Lénine que je reconnais dans Adorno et dans Lukacs est celui de la résolution du deuxième et du troisième congrès du Comintern sur le rôle du parti politique dans la révolution prolétaire. N'est-ce pas là un résumé d'une fausse histoire du parti bolchévique? Une histoire du parti bolchévique qui rétroprojette le caractère pris par le parti entre 1918 et 1921 dans des conditions de guerre civile sur la préhistoire du parti avant 1917 ?

Ensuite, pour Marx et Engels, de façon constante, des années 1840 jusqu'à la mort d'Engels, à l'exception d'une brève période lors de la Première Internationale quand ils ont fait alliance avec les proudhoniens, le problème, formulé dans la résolution du Congrès de La Hague en 1871, était ceci : «la classe ouvrière ne peut agir sauf en s'organisant en parti politique ». Comment les tentatives de rendre Marx plus hégélien expliquent-elles de façon satisfaisante cet aspect politique des interventions de Marx et d'Engels ?

CC : Peut-être la différence entre le Lénine que vous reconnaitriez et le Lénine officiel du Comintern est-elle la même différence que vous proposez ensuite entre Marx luimême (ou Marx et Engels) dans sa pratique politique, et le Marx « hégélienisé » qu'on trouve dans Lukács et dans Adorno.

Lénine a fait une contribution spécifique à l'histoire du marxisme qu'on ne peut ignorer, à savoir qu'il a été le grand schismatique du marxisme, il l'a divisé. C'est cela qui l'honore aux yeux d'Adorno. La vision de Lénine n'est pas celle d'une avant-garde minoritaire, mais celle d'une politique au sein de la classe ouvrière. Ce que Lénine introduit dans la Deuxième Internationale, c'est l'idée de partis ouvriers en concurrence, tous prétendant être anticapitalistes, révolutionnaires et marxistes. La crise du marxisme réfère aux controverses politiques au sein du marxisme. Nier cela, c'est prétendre que la politique se réduit à être simplement « les ouvriers contre les capitalistes ", et non un phénomène interne à la classe ouvrière. Le parti kautskyste, l'idée "d'une classe, un parti ", de l'intérêt unitaire des ouvriers vis-à-vis des capitalistes, de la volonté d'être le parti de la classe entière, tout cela nie que le contenu de l'émancipation politique peut être disputé parmi des ouvriers, et parmi des marxistes de partis différents. 
AF : Il me semble que la position prise par Lénine ne peut être facilement expliquée ou justifiée en termes de théorie marxiste, et que les écrits de Lukacs et de Gramsci sont une tentative de fonder cette pratique sur la théorie marxiste en trouvant «l'anneau manquant ». Il y a beaucoup d'affirmations chez Lénine, surtout dans ses écrits de jeunesse, qui ne font pas de théorie cohérente de sa pratique. Mais Lénine savait bien ce qu'il faisait, et sa pratique a eu une signification historique, comme vient de l'expliquer Chris. Alors, cette question peut être posée séparément de la réalité historique, et de tout jugement sur la pratique de Lénine par rapport à la théorie marxiste. Lukács a reconnu que Lénine avait accompli quelque chose d'important historiquement, et il a essayé de trouver le moyen de réviser et d'interpréter la théorie afin d'englober ce que Lénine avait fait. Lukács a fait une avancée théorique importante en comprenant comment il y aurait une connexion entre la classe ouvrière, la théorie marxiste, et les partis politiques qui représentent les ouvriers; comment il y aurait une connexion fondée dans une relation ontologique, une relation à la relation partagée à plusieurs niveaux, entre ces instances différentes du mouvement. C'est une idée théorique très importante, qu'on ne trouve pas, à mon avis, chez Marx et Engels ni chez Lénine. Elle est nécessaire pour comprendre ce qui s'est passé historiquement.

RW : Lukács veut que le parti, finalement, grandisse en mouvement de masse, c'est très clair. Mais en attendant, il dit explicitement dans son essai sur l'organisation du parti que chaque école différente, chaque avis différent sur la question même de ce que le parti doit faire, a besoin de se donner une forme organisationnelle. Il est tout à fait en faveur de l'éclosion générale, pluraliste de pratiques diverses, ce qui mine, à mon avis, l'idée d'un seul parti concentré d'avant-garde. On court le risque du sectarisme radical, mais dans la perspective de Lukács, au moins on évite la réification.

NB : Que Lukács et Adorno aient raison sur Lénine n'est pas la même question - il vaudrait mieux qu'elle en soit distincte - que celle de l'utilité politique de Lénine, autrement dit, que faire aujourd'hui ? On ne peut « hégélieniser » Marx, car Marx est déjà plus hégélien que Hegel!

QUESTION : Si je comprends bien, l'idée maîtresse de l'argument qu'Adorno soit un léniniste est de recruter cet Adorno léniniste pour le projet de reconstituer la gauche. Quelle est I'utilité de voir Adorno comme un léniniste?

CC : Adorno s'inscrit lui-même dans le projet léniniste. Il le dit : « Je veux être fidèle à Lénine ». Que veut-il dire par cette remarque ? 99,9 \% des léninistes dans le monde n'auraient pas accepté qu'Adorno fût fidèle à Lénine, de quelque manière que ce soit. Alors, je renverserais la question pour dire que je m'intéresse au Lénine qui devient visible à travers Adorno. Quand Adorno veut « un manifeste fortement léniniste », ce n'est pas contre Luxemberg. C'est une tentative lukácsienne de saisir ce que les éléments radicaux de la Deuxième Internationale avaient en commun. Pourquoi Luxemberg se disait-elle bolchévique ? Elle a écrit un essai dans les derniers mois de sa vie intitulé "Qu'est-ce le bolchévisme allemand?». Autrement dit : ce que nous voulons, c'est d'être du côté des bolchéviques. C'est une critique venant d'une camarade - c'est cela qui est important. Alors je m'intéresse à quoi ressemble l'histoire du marxisme, spécifiquement à travers les yeux d'Adorno, les yeux de Lukács, les yeux de Korsch; ce serait négligent de notre part d'ignorer les aperçus qu'ils ont eus de cette histoire. 
AF : À ce moment de l'histoire, nous connaissons si peu des forces d'opposition, de leur potentiel et de comment elles vont évoluer que nous n'aurons ni la base théorique ni la base en expérience pratique que le mouvement socialiste avait à l'époque de la formation et du développement des partis. Dans les conditions actuelles, il faut essayer d'identifier les sources d'opposition et les tensions autour du pouvoir réifiant des institutions, où qu'elles se trouvent, même si elles ne semblent pas politiques. Ce serait de fermer le ban prématurément que d'avoir une théorie et un parti qui essaient de diriger les luttes.

CC: Que veut-on dire par parti? D'une part, la formation d'un parti d'un type historiquement reconnaissable en ce moment actuel serait de verrouiller les possibilités. D'autre part, j'ai des réserves sur le moment Hardt-Negri actuel à l'égard du mouvementisme, qui voit le parti comme le chemin menant au stalinisme. Si nous disons que le mouvement socialiste d'autrefois avait de l'expérience historique accumulée, il faut alors constater que, pendant une génération, nous ne l'avons pas eu. Alors, on nous laisse répondre " quelque chose comme un parti » à la question de «forme». Ce que Richard nous indique à propos du concept de forme, c'est très important. Mais il y a danger à l'appliquer de façon trop générale dans ce que j’ai qualifié de "suivisme ", comme une justification de ce que nous faisons déjà. C'est un danger auquel je résisterais. Mais, je suis d'accord qu'il serait irréfléchi que d'essayer de fonder un parti d'après un modèle historique, condamné à être mort-né.

RW : La mémoire institutionnelle d'un parti est cruciale; à mon avis, son absence a mené à un effondrement désastreux de la pensée progressiste. C'est là où Lukacs a raison de critiquer Luxemburg, parce qu'un parti peut former cette mémoire institutionnelle.

Quant au point soulevé par Andrew, on ne sait pas vraiment quelles forces d'opposition existent. L'acte de former, ou de soutenir la formation d'un parti est une des façons pour le savoir. J'ai déjà parlé de l'insistance de Lukacs que chaque position devrait essayer de développer ses propres formes d'organisation. C'est comme ça qu'on peut le savoir. Si nous traitons cela comme une question purement sociologique, nous risquons de retomber dans la même optique réifiée d'un recueil de faits, plutôt que de s'engager dans la pratique. Encourager le développement de partis, de formes institutionnelles variées est une manière pour faire naître ces forces oppositionnelles. Sans cela, ces forces finissent par être moins cohérentes et moins conscientes de leur capacité.

QUESTION : Sans poussée vers la formation d'un parti, sans position forte sur le besoin d'un leadership, comment peut-on appliquer pratiquement ces diverses théories à la classe ouvrière ? Les conditions qui existaient dans les années 1920, 1930, 1950 n'existent plus aujourd'hui. Sans parti, sans leadership, quel espoir avons-nous?

RW : J'hésiterais à m'exprimer ainsi. Il est dangereux de parler d'appliquer des théories à la classe ouvrière. La question de leadership touche à cela. On y a déjà fait allusion, mais je pense que si le Tea Party a eu pas mal de succès, malgré ses incohérences et ses absurdités évidentes, c'est justement à cause de l'absence d'un leader et de la disponibilité de ses figures totémiques. Il y a des voix, mais il n'y a pas un seul leader, donc il existe plusieurs Tea Parties. Une des raisons qu'ils ont tant de succès, c'est qu'ils sont répandus, diffus et décentralisés. 
AF : Bien sûr, si nous avions un parti avec de l'autorité, et qui était écouté, nous serions en meilleure forme. Mais comment y arriver?

$\mathrm{CC}$ : Ce qui fonctionne pour la droite ne peut fonctionner pour la gauche. Il y a une différence fondamentale entre la droite et la gauche: la droite prospère sur l'incohérence, ce qui est interdit à la gauche. Je dirais aussi, de façon assez polémique, que les Tea Parties sont les vrais enfants de la Nouvelle Gauche.

L'idée de leadership théorique, dans le sens d'une théorie à appliquer, est précisément ce que la tradition marxiste voulait surmonter. C'est ce qu'elle comprenait comme une notion «bourgeoise » de théorie ou d'épistémologie. Remontant le fil jusqu'à Kant, cependant, il y avait déjà l'idée d'une pratique consciente de soi - il ne s'agit pas d'application abstraite de la théorie à la pratique. Il y a une continuité, je pense, entre Kant, Hegel et Marx; l'enjeu est d'essayer d'élever les pratiques existantes à la conscience de soi. Cela est tout à fait différent que de construire une théorie et de l'appliquer à la réalité.

Remarques en guise de conclusion

14 AF : Je pense que la gauche vit toujours sous l'horizon des demandes et des dissatisfactions qui ont émergé dans les années 1960 et 1970. Les mouvements écologistes, féministes, et d'autres formes de protestation qui sont apparues dans les secteurs spécifiques de la société comme la médecine viennent des catégories développées par la Nouvelle Gauche, afin d'articuler de nouveaux types de dissatisfaction. C'est cela, la contribution faite par Marcuse ; Adorno et Horkheimer n'y ont pas contribué, car ils ont perçu la Nouvelle Gauche comme un spot sur l'horizon plutôt mineur. Je suis en réalité extrêmement perplexe par l'éclipse de la pensée de Marcuse à gauche, et par la montée d'une nouvelle vision de l'École de Francfort centrée sur Benjamin, Adorno et Horkheimer. Pour moi, cela signifie un certain manque de sérieux politique qu'on puisse ignorer le seul théoricien qui s'était réellement engagé avec la sorte de politique à gauche dont on est capable aujourd'hui.

RW : J'aimerais conclure en répondant à ce «manque de sérieux politique ». La raison pour le retour des penseurs comme Adorno et Benjamin est que leur réception universitaire passe principalement par les départements de littérature ou de cultural studies. Je pense que la raison pour cela est précisément l'absence d'engagement ou d'actions directes. L'importance d'engagement et de quelque forme de pratique, sans oublier un certain niveau de leadership qui les accompagne sont cruciales.

CC : Je conclurai avec un plaidoyer pour qu'on prenne Adorno au sérieux comme un penseur politique, et non simplement un homme de lettres. Certes, il dit que « la musique et l'art sont ce que je connais, et donc ce sur quoi j'écris ». Mais il était là faussement modeste, car il a fait une intervention très forte dans la sociologie allemande sur la question de la modernité et du capital, introduisant la technique empirique de la sociologie américaine d'une part, et l'approche durkheimienne de l'autre ; ce, en opposition à l'approche wébérienne dominante. Dans sa correspondance avec Marcuse en 1969, marquée par l'amertume vis-à-vis de la controverse provoquée par la Nouvelle Gauche, Adorno dit à Marcuse : «Écoute, c'est l'Institut, notre vieil Institut, le même. » À quoi, Marcuse répond : «Tu sais aussi bien que moi combien la différence est grande entre le travail que menait l'Institut dans les années 1930 et son 
travail dans l'Allemagne actuelle. » Adorno ne peut que répondre: «Et quid de mes livres?» Autrement dit, quid des livres que l'existence de l'Institut m'a permis d'écrire? Adorno était le champion solitaire d'un marxisme hégélien au sein de la sociologie et de la philosophie allemandes. Ses œuvres sont des affirmations puissantes relayant les aperçus précieux de la tradition marxiste antérieure de Lukács et de Korsch dans le sillage de la crise du marxisme après les révolutions échouées du début $\mathrm{du} 20^{\mathrm{e}}$ siècle. Adorno a essayé de maintenir en vie cette tradition.

17 Ainsi, je défendrais Adorno contre ses dévoués actuels. L'Adorno qui prend son envol dans les humanités aujourd'hui est un Adorno édulcoré, dépolitisé, un Adorno dont le marxisme est filtré, ou bien transformé en critique éthique de la société. Je pense au contraire qu'Adorno a beaucoup à nous dire d'importance politique sur le problème de la théorie et de la pratique. 